\title{
On the translocation of proteins across membranes
}

(mitochondrial import/secretion/endoplasmic reticulum)

\author{
S. J. Singer, Pamela A. Maher, And Michael P. Yaffe \\ Department of Biology, University of California at San Diego, La Jolla, CA 92093
}

Contributed by S. J. Singer, October 22, 1986

\begin{abstract}
Many proteins of intracellular organelles are first synthesized in the cytoplasm and are then specifically transferred across the membranes of the organelles. On the assumption that these transfers all occur by the same basic mechanism, we enumerate the rather stringent requirements that the mechanism must satisfy. A unitary molecular mechanism is then proposed that meets these requirements.
\end{abstract}

In eukaryotic cells, a great variety of hydrophilic polypeptides synthesized in the cytoplasm are transferred across one, two, or three membranes to become incorporated into one of at least four different organelles: the endoplasmic reticulum (ER), mitochondria, chloroplasts, or peroxisomes. A similar process occurs during the externalization of many polypeptides synthesized inside bacteria. Such a transfer (termed translocation) must overcome formidable thermodynamic barriers involved in the movement of the ionic and polar residues of the hydrophilic polypeptide across the hydrophobic interior of the membrane. The molecular mechanisms for translocation are at present unknown. It seems reasonable that all such translocations might occur by a unitary mechanism that originated with prokaryotes and subsequently evolved and diversified in eukaryotes. On the other hand, until recently it was thought that in eukaryotes fundamentally different mechanisms operated in translocation across ER membranes and, for example, mitochondrial membranes (for review, see ref. 1). The evidence had suggested that translocation across ER membranes was obligatorily cotranslational-i.e., that translocation occurred only while the polypeptide chain was being synthesized on the ribosomewhereas with mitochondria it could be post-translationali.e., translocation could occur after the polypeptide was synthesized and released from the ribosome. In a recent report (2), however, we have provided evidence that the differences between translocation across ER and mitochondrial membranes were more apparent than real and reflected the in vitro formation of intrachain disulfide bonds in the ER-targeted polypeptides before their translocation.

In this paper, we assume that there is a unitary mechanism for all translocations of polypeptides across membranes, which, with relatively minor modifications, can accommodate the diversity of translocations known to exist. The molecular components, thermodynamic restrictions, energy requirements, and other factors that are likely to be involved in such a mechanism are first enumerated. [Some of this material has been considered earlier $(1,3)$, but from a different point of view.] We then suggest a mechanism that conforms to these stipulations and requirements.

Closely related to the problem of the complete translocation of a hydrophilic polypeptide across a membrane is the problem of the intercalation of integral proteins into membranes. This transfer of proteins into membranes will be taken up in a subsequent paper (4).

The publication costs of this article were defrayed in part by page charge payment. This article must therefore be hereby marked "advertisement" in accordance with 18 U.S.C. $\$ 1734$ solely to indicate this fact.

\section{Components of the Translocation Machinery}

There are two conceivable ways for translocation of a polypeptide across a membrane to occur. One is directly through the lipid bilayer, and the other is by means of some integral protein translocation apparatus (3). It has been variously suggested that polypeptides might insert spontaneously into the lipid bilayer and with some suitable energy input could then be directly transferred across (cf. refs. 1, 5, and 6). All such mechanisms, in our opinion, have not properly addressed the thermodynamic problem of the transfer of ionic residues of hydrophilic polypeptides through the nonpolar interior of the bilayer.

An early quantitative analysis of the thermodynamics of membrane systems (7) indicated that it is energetically very costly to remove ionic residues from water and insert them into a medium of low dielectric constant such as the interior of the lipid bilayer $(7,8)$. Among the distinctive features of the present paper is an insistence on the requirement that the ionic groups of polypeptides must largely remain in contact with water throughout the process of translocation of the hydrophilic chain across the hydrophobic interior of the membrane, in order that the free energy of activation of the translocation process be sufficiently small.

It has recently been demonstrated in some cases that signal peptides (see below) can themselves bind to lipid bilayers (9-13). The capacity of these oligopeptides to form amphipathic $\alpha$-helices has been prominently mentioned as involved in such lipid binding. We suggest that in such cases the structure formed in the membrane is usually a transmembrane aggregate of several chains of the oligopeptide forming an aqueous channel through the membrane with all of the ionic residues facing into the channel as in the cases of mellitin $(14,15)$ and alamethicin (16). Such channel formation could account for the fact that at appropriate concentrations synthetic signal peptides can uncouple respiratory control of mitochondria (9), presumably by obliterating the proton gradient across the mitochondrial membrane. In any event, we think that it is unlikely that such multimolecular binding of signal sequences to lipid bilayers has anything directly to do with the mechanism of translocation; for one thing, translocation is most likely a monomolecular process (see below).

The requirement that the great majority of the ionic residues of the polypeptide must remain in contact with water while they are being transferred across the membrane is consistent with experimental evidence (17) that the polypeptide chain is accessible to aqueous media during translocation. This criterion makes it exceedingly unlikely that translocation of a hydrophilic polypeptide occurs directly across the lipid bilayer. The only alternative is that proteins integral to the membrane must be involved in translocation.

Translocator Proteins. The process of importing a polypeptide from the cytoplasm into an organelle can be divided

Abbreviations: ER, endoplasmic reticulum; TP, translocator protein. 
conceptually into two aspects, targeting and translocation. Targeting ensures that a polypeptide is directed specifically to its appropriate organelle, and it presumably involves a mechanism for binding or docking $(18,19)$ the polypeptide from the cytoplasm to the specific organellar membrane. Translocation is the process of the transfer of the docked polypeptide across one or two membranes of the organelle. This latter process, as we have concluded in the previous section, most likely is mediated by special integral proteins in the membrane. We designate these as translocator proteins (TPs). It is unclear at present whether the TPs are involved in the targeting process as well as in translocation, or whether the targeting apparatus consists of proteins that are entirely unrelated to the TPs. Although this uncertainty exists, it is not of immediate concern in this article. Our primary focus is the translocation process itself and the proposal that TPs are required for translocation. In a later section, we describe a structural model for TPs that can satisfactorily account for their role in translocation and that leaves moot the question of their role in targeting.

Signal Sequences. Polypeptides to be translocated across membranes generally contain a stretch of amino acid sequence within the first approximately 60 residues from the amino terminus called the signal sequence. If this sequence is removed from the polypeptide, or if certain residues are deleted or mutated $(20,21)$, translocation is abrogated. Furthermore, the replacement by genetic engineering methods of a signal sequence on a polypeptide chain by a different one is sufficient to target the polypeptide to the organelle appropriate to the new signal sequence (22). Aside from certain conserved features, however, remarkably wide variability exists in the amino acid sequence in the signal region for targeting to a single membrane type (e.g., bacterial membranes; see ref. 23 for review). This variability must far exceed the number of different TPs in a given membrane. This implies, therefore, that the binding of a signal sequence to a TP, unlike the binding of a substrate to an enzyme, which involves close complementarity, likely involves a weak interaction that invokes certain secondary structural features common to a range of signal sequences.

There appear to be two major classes of signal sequences, superimposed upon the heterogeneity just discussed. One class (which we term hydrophobic) contains a central hydrophobic region (24), whereas the second class (termed hydrophilic) does not (25). A unitary model of translocation must be able to accommodate the existence of the two widely different classes of signal sequences and of the pronounced heterogeneity encountered within each of them.

The translocation of many polypeptides is accompanied or followed closely by proteolytic cleavage of their signal sequences at their carboxyl termini on the trans side of the membrane. (We designate the cytoplasmic side of a membrane as cis, the other side as trans.) This proteolytic processing is apparently not required for translocation (26-28); it may generally be required, however, for the efficient release of many soluble proteins from their association with the membrane after translocation $(27,29)$.

Postsignal Sequences. For hydrophilic polypeptides that, apart from their cleaved signal sequence, are completely translocated across membranes, the amino acid sequences that follow the signal sequence do not appear to play a discriminatory role in translocation or in targeting the polypeptide to a particular organelle. This has been dramatically demonstrated by gene-fusion experiments in which signal sequences were added to the amino termini of proteins that are normally cytoplasmic. Such hybrid proteins were imported into the organelle appropriate to the signal sequence $(30-34)$. The clear inference is that once the signal sequence of a polypeptide, in conjunction with the appropriate TP, initiates the process of translocation, almost any postsignal hydrophilic amino acid sequence of any total length can be translocated.

Energy Utilization in Translocation. For most translocatory systems that have been studied in sufficient detail, translocation will not occur in the absence of a suitable energy source. For different systems, however, the nature of the energy source can vary [e.g., an electrochemical gradient across mitochondrial membranes $(35,36)$ or ATP for ER membranes (37-39)]. In some cases, the energy for translocation appears to be derived from the attachment of a ligand to the polypeptide on the trans side of the membrane, as in the covalent attachment of the heme during translocation of apocytochrome $c$ across the outer mitochondrial membrane (40). Precisely how energy is utilized in any translocation process is unknown. Energy might be required only for the initiation of translocation and then might not be required for the transfer of the rest of the polypeptide chain across the membrane (41). On the other hand, energy input may be required throughout the entire process of translocation.

\section{Assumptions and Restrictions of a Unitary Mechanism}

Co-Translational and Post-Translational Translocation. With ER (2, 37-39) and mitochondrial (42) systems, it has been demonstrated that the translocation of a given polypeptide chain can generally occur either co-translationally or post-translationally. We assume that this is true for translocations in general. We also assume that all translocations, whether co-translational or post-translational, occur by the same mechanism (although possibly at different rates; see below). These assumptions carry several implications. One is that the residual attachment of a nascent polypeptide chain to the ribosome during co-translational translocation cannot be a necessary feature of the translocation mechanism (5). Another implication is that translocation is almost certainly always monomolecular in the polypeptide to be translocated, since it is difficult, in view of the proximity of a massive ribosome, to envision more than one polypeptide chain being involved in a single co-translational translocation event. In other words, concerted mechanisms, in which two or more polypeptide chains undergo translocation together, seem unlikely. A third implication is that after a signal sequencecontaining polypeptide chain is released from a ribosome and folds up in the aqueous solution into some three-dimensional conformation, the signal peptide must remain accessible for binding to the TP in the membrane to initiate post-translational translocation.

Translocation Occurs by Segments of the Polypeptide Chain. There are three modes by which a polypeptide chain could, in principle, be translocated across a membrane: $(i)$ the chain, starting at one end, could be continuously threaded through the membrane; (ii) the entire molecule might be translocated at once; or (iii) successive segments of the chain could be translocated as more or less discrete units one after the other. One set of experiments that casts doubt on a threading mechanism was performed by Coleman et al. (43). They showed that the same internal hydrophobic sequence of a polypeptide chain is treated differently during translocation, depending on its position within the chain (functioning in one case as an internal signal sequence and in the other as a stop-transfer sequence), a result that is incompatible with simple chain threading. Other types of experiments indicate that a secretory polypeptide, after signal peptide insertion and cleavage, is not translocated all at once; part of a chain can be detected on the trans side of the membrane while the remainder is still on the cis side $(44,45)$.

These results, by elimination, suggest that translocation of a polypeptide chain occurs by segmental units. Furthermore, a segmental mode is consistent with results $(2,46,47)$ demonstrating that stabilizing the three-dimensional confor- 
mation of the free prepolypeptide, so that unfolding of the chain is inhibited, abrogates post-translational translocation.

Segmental Translocation Occurs by Subdomains. Short oligopeptides ( $\approx 10-30$ amino acid residues) generally do not exhibit secondary structure in aqueous solutions; that is, they exhibit properties similar to those of random-coil polymers $(48,49)$. However, such sequences can exhibit secondary structure (forming a "subdomain") either by interaction with an adjacent short linear sequence of the same polypeptide chain or by interaction with a separate oligopeptide or protein surface $(48,49)$. In the latter case, it is said that secondary structure formation of the formerly structureless oligopeptide is "seeded" by the interaction. However, the secondary structure that is achieved in a subdomain need not necessarily be a unique one; a number of such structures may be of closely similar free energies (50). Because the secondary structure forms spontaneously upon seeding, the process must involve a decrease in free energy (i.e., energy is released).

We propose that polypeptides that are segmentally translocated across membranes do so by the formation and translocation of successive small subdomains of secondary structure, starting from the end of the chain bearing the signal sequence. Each subdomain in turn is formed (seeded) by interaction with the TP in the membrane and is then translocated with the utilization of energy. After all of the polypeptide has been translocated, the final equilibrium conformation of the protein in aqueous solution is attained.

\section{A Unitary Mechanism for Translocation}

We next present the outlines of a unitary mechanism for translocation that accommodates the facts, restrictions, and assumptions that have been discussed. In the mechanism proposed, translocator proteins are assigned a specific structural organization in the membrane, which enables them to carry out the following: (i) the binding of a signal sequence on a polypeptide chain; (ii) the seeding of the formation of successive subdomains of the chain within the TP; (iii) the energy-dependent translocation of these subdomains one after the other until the entire chain is translocated; throughout this entire process, the ionic residues of the chain remain in contact with water.

Structures and Properties of Translocator Proteins. The crucial component of the translocation process is the TP. Blobel (3) has proposed that the TP may function as a transmembrane channel, but he has not presented a model of its structure or the detailed mechanism of translocation. We propose that TPs are integral proteins that are subunit aggregates consisting of $n$ homologous but not identical transmembrane subunits that together form a transmembrane aqueous channel down the central axis of the aggregate. The acetylcholine receptor aggregate (51) provides a precedent for such a structure. For reasons that will be given below, it is suggested that for TPs $n$ might generally lie between 3 and 6. That an individual TP functions in the specific transport of small hydrophilic molecules or ions through its central aqueous channel, in addition to serving in polypeptide translocation, is neither required nor ruled out by the model we propose, but it is an interesting possibility to consider.

The special feature we ascribe to all TP subunit aggregates is that at one of the $n$ nonidentical interfaces between neighboring subunits the two subunits are only weakly bound. Consider the TP structure represented schematically in Fig. 1 , where $n=4$. Suppose that only a relatively small energy input is required to separate the $\beta$ and $\gamma$ subunits. This interface provides the passageway for the translocation process.

Translocation of a single polypeptide chain is initiated by the binding of a part of the signal sequence to the $\beta$ or $\gamma$
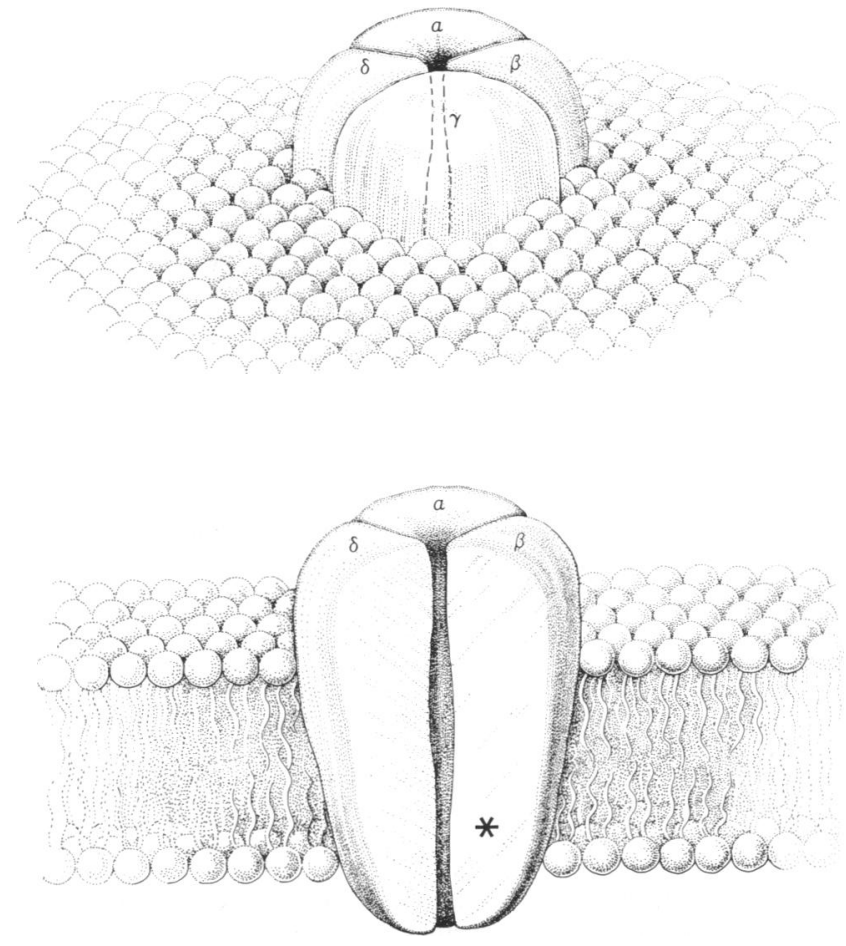

FIG. 1. Schematic representation of the proposed structure of a translocator protein, here depicted (Upper) as a subunit aggregate with four nonidentical but homologous subunits surrounding an aqueous channel through the membrane. The $\gamma$ subunit has been removed (Lower) to reveal part of the interface between the $\beta$ and $\gamma$ subunits. The asterisk designates a site in the interface described in Fig. 2.

subunit, where it is exposed at the cis face of the membrane, and the intercalation of the rest of the signal sequence at the interface between the $\beta$ and $\gamma$ subunits. The intercalation of the signal sequence, mediated by its interactions with sites within the interface, is such as to position the cleavable bond following the signal peptide at the trans face of the membrane. This requires the intercalation of the next sequence of $\approx 20-30$ amino acids into the interface, which, either with or without the direct involvement of the signal sequence, forms a secondary structure (a subdomain) within the $\beta-\gamma$ interface that is seeded by interaction of the intercalated peptide with those TP subunits (Fig. 2 Left). The signal sequence and the first subdomain that are intercalated in the TP are proposed to exhibit the following structural features: (i) all of the charged groups of their ionic residues are positioned into the aqueous channel of the TP, to satisfy hydrophilic interactions; (ii) their nonpolar residues are generally positioned within the $\beta-\gamma$ interface away from contact with water; (iii) the intercalated structure exposes the cleavable bond following the signal sequence to the action of the cleavage protease on the trans side of the membrane; and (iv) the secondary structure of the subdomain need not be unique (50), as long as it develops a sufficient but weak affinity for the $\beta-\gamma$ interface; the secondary structure could contain a stretch of amphipathic helix, a $\beta$-structure folded into the interface, or both.

The energy required to separate the $\beta$ and $\gamma$ subunits at their interface could be supplied from some external source [e.g., ATP in the case of the ER (37-39)]. On the other hand, the energy could be supplied by the binding of the signal sequence to the TP, as well as from the formation of the secondary structure of the first subdomain and its binding within the $\beta-\gamma$ interface. The number of amino acid residues in a subdomain could vary to some extent but might be limited 


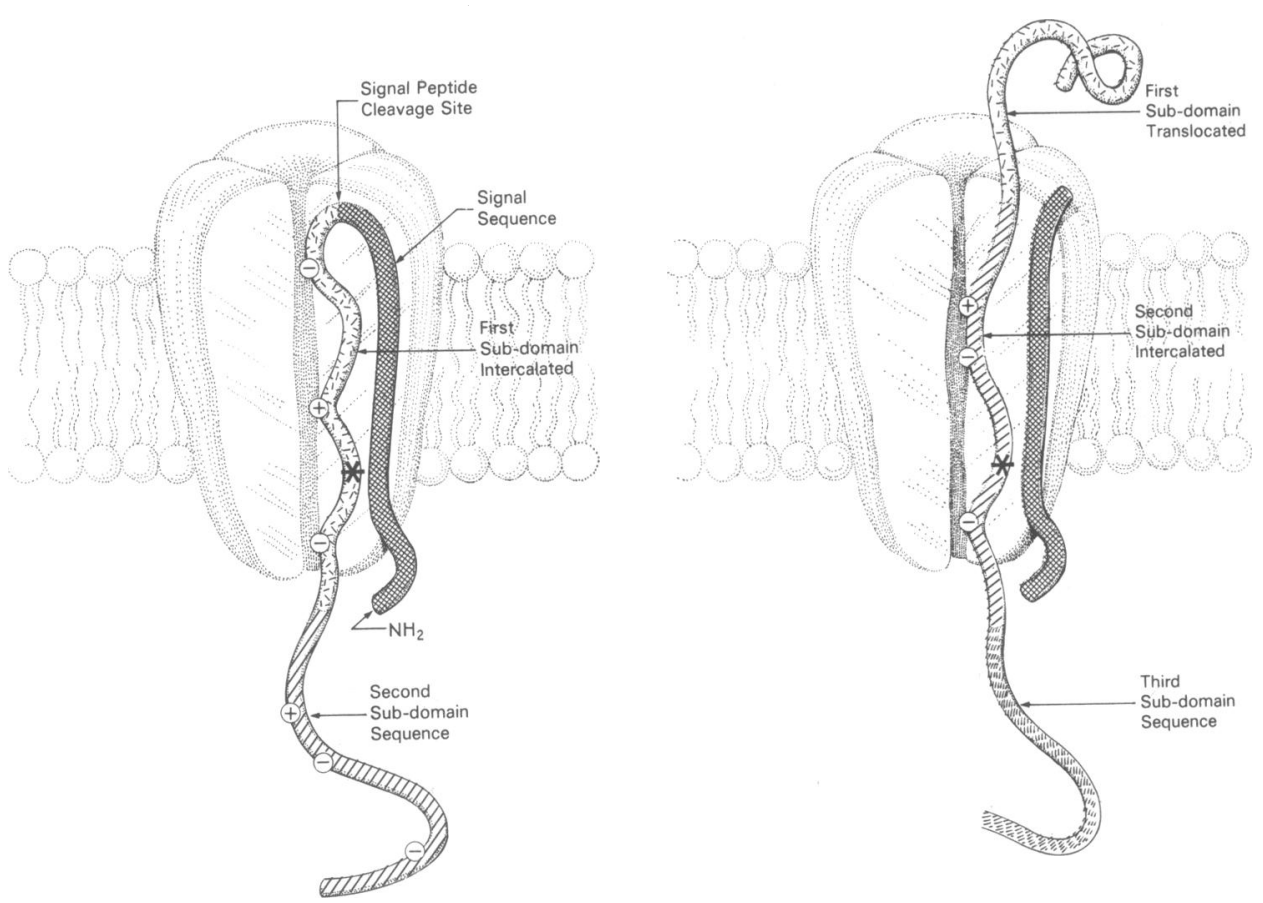

Fig. 2. Schematic representation of two stages in the proposed model for the translocation of a hydrophilic prepolypeptide via the $\beta-\gamma$ interface of the translocator protein shown in Fig. 1. In the stage shown at the left, the signal sequence (of the hydrophobic class) is shown intercalated into the interface, with its cleavage site properly oriented to the trans face of the membrane, and the immediately following sequence of $\approx 20-40$ hydrophilic residues forming the first subdomain is shown bound within the interface, with its ionic residues facing into the aqueous channel of the translocator aggregate. In a later stage shown at the right, the signal peptide has been cleaved but is depicted as remaining bound at the $\beta-\gamma$ interface; the first subdomain has been translocated, resulting in the intercalation and formation of the second subdomain in the interface. The asterisk denotes a particular site on the $\beta$ subunit in the interface that might be critical to the interaction with a residue on each successive subdomain. See text for further details.

to $\approx 20-40$ residues by the accessibility and area of the $\beta-\gamma$ interface and the secondary structure of the subdomain within the interface. If the number of subunits, $n$, is large enough, the strain on the other subunits arising from the intercalation of the signal peptide and first subdomain between $\beta$ and $\gamma$ would be small. On the other hand if $n$ were too large, the central aqueous channel might have too large a diameter to restrict small molecule transport. The choice of $3 \leq n \leq 6$ is arbitrary but is meant to meet these requirements. With regard to the existence of the two major classes of signal sequences discussed above, two different classes of TP, exhibiting somewhat different characteristic $\beta$ and $\gamma$ subunits and interfaces, could be invoked to accommodate them structurally. A TP for the hydrophobic class would bind the hydrophobic portion of the sequence at the $\beta-\gamma$ interface in an area removed from the aqueous channel (as depicted in Fig. 2 Left), whereas a TP for the hydrophilic class would bind the signal sequence near the aqueous channel.

Translocation of the first subdomain would probably be an energy-requiring step. Translocation could be accomplished, for example, by energy-induced conformational changes in the $\beta$ and/or $\gamma$ subunits which could produce extensive quaternary rearrangements of the TP $(7,52,53){ }^{*}$ These

\footnotetext{
*An important feature of subunit aggregates is their capacity to undergo large quaternary rearrangements (7) of their subunits with a relatively small input of energy. The large relative displacement of the $\alpha$ and $\beta$ subunits of the hemoglobin molecule upon binding oxygen (52) and the closure of the gap junction connexon upon binding $\mathrm{Ca}^{2+}(53)$ are examples of such quaternary rearrangements. In the case of TPs, occupancy of the $\beta$ - $\gamma$ interface by an intercalated subdomain could result in a conformational change that, for example, rendered the TP susceptible to modification by a kinase [in cases in which ATP is utilized as the source of translocation energy (39-41)]; the phosphorylation might then power the quaternary rearrangement of the TP and the displacement of the subdomain to the trans side.
}

would serve to displace the first subdomain from within the $\beta-\gamma$ interface into the aqueous phase on the trans side of the membrane. In the process of translocation of the first domain, the immediately following sequence (the initial $\approx 10$ residues of the second subdomain) of the polypeptide chain would be "pulled into" the $\beta-\gamma$ interface and this would help serve to keep the interface open. A cleavable signal peptide might not be cleaved until later in the overall translocation process, or it might be cleaved and remain in the interface after the translocation of the first subdomain (as depicted in Fig. 2 Right). Alternatively, the signal sequence might not be cleaved at all. The retention of the signal sequence in either case would also help keep the interface open. The open $\beta-\gamma$ interface would permit seeding of the second subdomain within the interface-i.e., permit the additional intercalation of the next $\approx 20$ residues of the chain from the cis side of the membrane so as to form the second subdomain (Fig. 2 Right). The rules for the positioning of the amino acid residues of the second subdomain within the $\beta-\gamma$ interface would be the same as for the first subdomain: ionic residues facing into the aqueous channel, etc. The displacement of the second subdomain into the aqueous phase on the trans side of the membrane would then follow by a mechanism similar to that used to displace the first, except that part of the energy required might be derived from conformational interactions between the two translocated domains. This process of successive subdomain translocation $(\approx 20-40$ residues at a time) would continue until the entire polypeptide chain was translocated across the membrane.

This mechanism should allow for the accommodation and translocation of any of a wide range of sequences of generally hydrophilic polypeptides, because there is no specific or entirely fixed conformation required for a subdomain to be intercalated into the $\beta-\gamma$ interface, and only a few rules for the appropriate positioning of the amino acid residues of an intercalated subdomain have to be satisfied. The interaction 
of a subdomain with the $\beta$ and $\gamma$ subunits at the interface must be weak enough to allow the subdomain to dissociate readily during its translocation. These considerations would account for the capacity of the translocation mechanism to transfer a wide range of generally hydrophilic postsignal sequences. If the signal sequence of a polypeptide chain was not cleaved and did not of itself have too strong an affinity for the TP, then, after the bulk of the polypeptide chain was translocated, the signal sequence might form part of the last subdomain of the chain and thus be translocated with it and released to the trans side of the membrane. This could account for the complete translocation of certain signal-retaining polypeptides $(54,55)$. An uncleaved signal peptide under other circumstances could anchor a polypeptide chain in the membrane (4).

This entire translocation mechanism could operate either co-translationally or post-translationally. In the former case, the intercalation and translocation of successive subdomains of the polypeptide chain would occur as successively more carboxyl-terminal portions of the chain were synthesized on the ribosome. If, however, the polypeptide chain were first completed and released from the ribosome into the aqueous solution on the cis side of the membrane, the chain would probably rapidly fold up into a three-dimensional conformation (2) in which the signal sequence would have to be exposed or easily accessible. Post-translational translocation would then involve the successive unfolding of the polypeptide chain to permit the intercalation of the signal sequence and successive subdomains of the polypeptide sequence into the $\beta-\gamma$ interface and the sequential translocation of the subdomains. The unfolding of the completed chain might be rate limiting, and as a result post-translational translocation of a polypeptide chain might take a longer time than its co-translational translocation $(56,57)$.

We are grateful to Dr. Russell F. Doolittle for his continued interest and helpful discussions. Mrs. Myrtali Anagnostopoulos created Figs. 1 and 2 to grace the text.

1. Wickner, W. T. \& Lodish, H. F. (1985) Science 230, 400-407.

2. Maher, P. A. \& Singer, S. J. (1986) Proc. Natl. Acad. Sci. USA 83, 9001-9005.

3. Blobel, G. (1980) Proc. Natl. Acad. Sci. USA 77, 1496-1500.

4. Singer, S. J., Maher, P. A. \& Yaffe, M. P. (1987) Proc. Natl. Acad. Sci. USA 84, in press.

5. von Heijne, G. \& Blomberg, C. (1979) Eur. J. Biochem. 97, 175-181.

6. Engelman, D. M. \& Steitz, T. A. (1981) Cell 23, 411-422.

7. Singer, S. J. (1971) in Structure and Function of Biological Membranes, ed. Rothfield, L. I. (Academic, New York), pp. 145-222.

8. Singer, S. J. (1976) in Structure of Biological Membranes, eds. Abrahamsson, S. \& Pascher, I. (Plenum, New York), pp. 443-461.

9. Roise, D., Horvath, S. J., Tomich, J. M., Richards, J. H. \& Schatz, G. (1986) EMBO J. 5, 1327-1334.

10. Briggs, M. S. \& Gierasch, L. M. (1984) Biochemistry 23, 3111-3114.

11. Briggs, M. S., Gierasch, L. M., Zlotnick, A., Lear, J. D. \& DeGrado, W. F. (1985) Science 228, 1096-1098.

12. Briggs, M. S., Cornell, D. G., Dluhy, R. A. \& Gierasch, L. M. (1986) Science 233, 206-208.

13. Ito, A., Ogishima, T., Ou, W., Omura, T., Aoyagi, H., Lee, S., Mihara, H. \& Izumiya, N. (1985) J. Biochem. 98, 1571-1582.

14. Tosteson, M. T. \& Tosteson, D. (1981) Biophys. J. 36, 109-116.

15. Eisenberg, D. (1984) Annu. Rev. Biochem. 53, 595-623.
16. Fox, R. O. \& Richards, F. M. (1982) Nature (London) 300 , 325-330.

17. Gilmore, R. \& Blobel, G. (1985) Cell 42, 497-505.

18. Walter, G. \& Blobel, G. (1981) J. Cell Biol. 91, 557-561.

19. Meyer, D. I., Krause, E. \& Dobberstein, B. (1982) Nature (London) 297, 647-650.

20. Silhavy, T. J., Benson, S. A. \& Emr, S. D. (1983) Microbiol. Rev. 47, 313-334.

21. Horwich, A., Kalousek, F., Fenton, W., Pollock, R. \& Rosenberg, L. (1986) Cell 44, 451-459.

22. Emr, S., Vassarotti, A., Garret, J., Geller, B., Takeda, M. \& Douglas, M. (1986) J. Cell Biol. 102, 523-533.

23. von Heijne, G. (1986) Nucleic Acids Res. 14, 4683-4690.

24. von Heijne, G. (1985) J. Mol. Biol. 184, 99-105.

25. von Heijne, G. (1986) EMBO J. 5, 1335-1342.

26. Schauer, I., Emr, S., Gross, C. \& Schekman, R. (1985) J. Cell Biol. 100, 1664-1675.

27. Dalbey, R. E. \& Wickner, W. (1985) J. Biol. Chem. 260, 15925-15931.

28. Yaffe, M. P., Ohta, S. \& Schatz, G. (1985) EMBO J. 4, 2069-2074.

29. Hurt, E. C., Pesold-Hurt, B., Suda, K., Oppliger, W. \& Schatz, G. (1985) EMBO J. 4, 2061-2068.

30. Lingappa, V. R., Chaidez, J., Yost, C. S. \& Hedgpeth, J. (1984) Proc. Natl. Acad. Sci. USA 81, 456-460.

31. Hurt, E. C., Müller, U. \& Schatz, G. (1985) EMBO J. 4, 3509-3518.

32. Hurt, E. C., Soltanifar, N., Goldschmidt-Clermont, M., Rochaix, J.-D. \& Schatz, G. (1986) EMBO J. 5, 1343-1350.

33. Carlson, M. \& Botstein, B. (1982) Cell 28, 145-154.

34. van Loon, A. P. G. M. \& Young, E. T. (1986) EMBO J. 5, 161-165.

35. Gasser, S. M., Daum, G. \& Schatz, G. (1982) J. Biol. Chem. 257, 13034-13041.

36. Schleyer, M., Schmidt, B. \& Neupert, W. (1982) Eur. J. Biochem. 125, 109-116.

37. Perara, E., Rothman, R. E. \& Lingappa, V. R. (1986) Science 232, 348-352.

38. Rothblatt, J. A. \& Meyer, D. I. (1986) EMBO J. 5, 1031-1036.

39. Waters, M. G. \& Blobel, G. (1986) J. Cell Biol. 102, 1543-1550.

40. Hennig, B. \& Neupert, W. (1981) Eur. J. Biochem. 121, 203-212.

41. Schleyer, M. \& Neupert, W. (1985) Cell 43, 339-350.

42. Hay, R., Böhni, P. \& Gasser, S. (1984) Biochim. Biophys. Acta 779, 65-87.

43. Coleman, J., Inukai, M. \& Inouye, M. (1985) Cell 43, 351-360.

44. Bergman, L. W. \& Kuehl, W. M. (1979) J. Biol. Chem. 254, 8869-8876.

45. Randall, L. L. (1983) Cell 33, 231-240.

46. Eilers, M. \& Schatz, G. (1986) Nature (London) 322, 228-232.

47. Randall, L. L. \& Hardy, S. J. S. (1986) Cell 46, 921-928.

48. Kim, P. S. \& Baldwin, R. L. (1985) Annu. Rev. Biochem. 51, 459-489.

49. Baldwin, R. L. (1986) Trends Biochem. Sci. 11, 6-9.

50. Harrison, S. C. \& Durbin, R. (1985) Proc. Natl. Acad. Sci. USA 82, 4028-4030.

51. Anholt, R., Lindstrom, J. \& Montal, M. (1984) in Enzymes of Biological Membranes, ed. Martinosi, A. (Plenum, New York), Vol. 3, 2nd Ed., pp. 335-401.

52. Perutz, M. F. (1969) Proc. R. Soc. London Ser. B. 173, 113-140.

53. Unwin, P. N. T. \& Ennis, P. D. (1984) Nature (London) 307, 609-613.

54. Meek, R. L., Walsh, K. A. \& Palmiter, R. D. (1982) J. Biol. Chem. 257, 12245-12251.

55. Braell, W. A. \& Lodish, H. F. (1982) J. Biol. Chem. 257, 4578-4582.

56. Reid, G. A. \& Schatz, G. (1982) J. Biol. Chem. 257, 13056-13061.

57. Reid, G. A. \& Schatz, G. (1982) J. Biol. Chem. 257, 13062-13067. 